\title{
Construction of global solutions for a symmetric system of Keyfitz-Kranzer type with three piecewise constant states
}

\author{
Pengyan Wang ${ }^{1}$, Chun Shen ${ }^{1 *}$ (D) and Xiuli Lin²
}

\section{"Correspondence:}

shenchun3641@sina.com 'School of Mathematics and Statistics Science, Ludong University, Yantai, P.R. China Full list of author information is available at the end of the article

\begin{abstract}
The exact solutions of the Riemann problem for a symmetric system of Keyfitz-Kranzer type are obtained in fully explicit forms. Furthermore, the global solutions of the double Riemann problems are also constructed explicitly when the initial data are taken to be three piecewise constant states. During the process of constructing the global solutions, all occurring wave interactions have been dealt with in detail by using the method of characteristics. In addition, it is shown that the Riemann solutions are stable with respect to the specific small perturbations of the Riemann initial data.
\end{abstract}

MSC: $35 \mathrm{LL} 65 ; 35 \mathrm{~L} 67 ; 76 \mathrm{~N} 15$

Keywords: Riemann problem; Wave interaction; Symmetric system; Keyfitz-Kranzer type; Temple class; Hyperbolic conservation law

\section{Introduction}

In this paper, we are concerned with the following hyperbolic system of conservation laws in the form

$$
\left\{\begin{array}{l}
u_{t}+\left(u \phi\left(u^{n}+v^{n}\right)\right)_{x}=0, \\
v_{t}+\left(v \phi\left(u^{n}+v^{n}\right)\right)_{x}=0,
\end{array}\right.
$$

which is named a symmetric system of Keyfitz-Kranzer type [1-5]. For convenience, we introduce the notation $r=u^{n}+v^{n}$. In the present work, we restrict ourselves only to considering the situation $\phi^{\prime}(r)>0$ and $\phi^{\prime \prime}(r)>0$ in the quarter $(u, v)$ phase plane, where $u \geq 0$ and $v \geq 0$ are required. It is easily shown that system (1.1) has two real eigenvalues $\lambda_{1}=\phi(r)$ and $\lambda_{2}=\phi(r)+n r \phi^{\prime}(r)$. Obviously, we have $\lambda_{1}<\lambda_{2}$ under our assumption $\phi^{\prime}(r)>0$ when $r>0$, which implies that system (1.1) is strictly hyperbolic except for the origin in the quarter $(u, v)$ phase plane. It is worthwhile to notice that the shock curve has the same expression formula as the rarefaction one in the quarter $(u, v)$ phase plane, so that system (1.1) belongs to the so-called Temple class [6, 7].

(c) The Author(s) 2019. This article is distributed under the terms of the Creative Commons Attribution 4.0 International License (http://creativecommons.org/licenses/by/4.0/), which permits unrestricted use, distribution, and reproduction in any medium, provided you give appropriate credit to the original author(s) and the source, provide a link to the Creative Commons license, and indicate if changes were made. 
It is easily seen that system (1.1) is a special situation for the following general KeyfitzKranzer system [8]:

$$
\left\{\begin{array}{l}
u_{t}+(u \phi(u, v))_{x}=0 \\
v_{t}+(v \phi(u, v))_{x}=0
\end{array}\right.
$$

The general Keyfitz-Kranzer system (1.2) was used as a tensile elastic model to describe the propagation of longitudinal and transverse waves [8]. It was also used in [9] to illustrate some features of the solar wind in magnetohydrodynamic. On the one hand, if $n=1$ is taken, then system (1.1) is simplified into

$$
\left\{\begin{array}{l}
u_{t}+(u \phi(u+v))_{x}=0 \\
v_{t}+(v \phi(u+v))_{x}=0
\end{array}\right.
$$

It is remarkable that the system of multi-component chromatography is just a special case of system (1.3) and thus was also studied for example in [10-15]. On the other hand, if $n=2$ is taken, then system (1.1) turns out to be

$$
\left\{\begin{array}{l}
u_{t}+\left(u \phi\left(u^{2}+v^{2}\right)\right)_{x}=0, \\
v_{t}+\left(v \phi\left(u^{2}+v^{2}\right)\right)_{x}=0 .
\end{array}\right.
$$

This symmetric Keyfitz-Kranzer system (1.4) has been widely investigated in [1, 2, 5, 8, 9], in which some peculiar phenomena were shown such as the propagation and cancelation of initial oscillations. In addition, it should be pointed out that the following asymmetric Keyfitz-Kranzer system

$$
\left\{\begin{array}{l}
\rho_{t}+\left(\rho \phi\left(\rho, u_{1}, u_{2}, \ldots, u_{n}\right)\right)_{x}=0, \\
\left(\rho u_{i}\right)_{t}+\left(\rho u_{i} \phi\left(\rho, u_{1}, u_{2}, \ldots, u_{n}\right)\right)_{x}=0,
\end{array}\right.
$$

was also proposed by Lu [16, 17], in which $i=1,2, \ldots, n$. It should be stressed that some well-known hyperbolic systems, such as the pressureless gas dynamics system $[18,19]$, the isentropic Chaplygin gas dynamics system [20-24], and the macroscopic production model [25], are recovered by choosing suitable $\phi$.

One can see from the above description that system (1.1) has been extensively considered when $n=1$ or $n=2$ is taken. However, there are relatively less studies on system (1.1) when $n$ is the other number. It was shown in [5] that the existence of a weak entropy solution to the Cauchy problem for system (1.1) with bounded measurable initial data was obtained by using the vanishing viscosity approach when $n>1$ was taken. Based on the above result, the present work under consideration is devoted to dealing with the special initial value problem for system (1.1) with $n>1$ subject to the following three piecewise constant initial data:

$$
(u, v)(x, 0)= \begin{cases}\left(u_{-}, v_{-}\right), & -\infty<x<0, \\ \left(u_{m}, v_{m}\right), & 0<x<x_{0}, \\ \left(u_{+}, v_{+}\right), & x_{0}<x<+\infty,\end{cases}
$$


where $x_{0}$ is a sufficiently small positive number. The initial data in the form (1.6) have been intensively used to investigate the interaction problem of elementary waves [26-32] for various hyperbolic systems of conservation laws. It is worth mentioning that the three piecewise constant initial data (1.6) are also taken to be a special small perturbation of the corresponding Riemann initial data

$$
(u, v)(x, 0)= \begin{cases}\left(u_{-}, v_{-}\right), & -\infty<x<0 \\ \left(u_{+}, v_{+}\right), & 0<x<+\infty\end{cases}
$$

Thus, the special initial value problem (1.1) and (1.6) is often named a double Riemann problem (or the perturbed Riemann problem) in the literature.

In the present paper, we construct the global solutions to the double Riemann problem (1.1) and (1.6) for all the possible situations under the assumptions $\phi^{\prime}(r)>0$ and $\phi^{\prime \prime}(r)>0$ when the initial data (1.6) lie in the quarter $(u, v)$ phase plane. During the process of constructing the global solutions, the wave interactions have been investigated intensively by using the method of characteristics [26,33]. First of all, we need to deal with the collision of waves belonging to different families. More precisely, we must consider the situation that the shock (or rarefaction) wave collides with the contact discontinuity. Consequently, we need to deal with the coalescence and cancelation of waves belonging to the same family. More specifically, we are concerned with the situation that the shock wave cancels the rarefaction wave or with the situation that the two shock waves coalesce into a new shock wave. In the end, it is shown that the limits $x_{0} \rightarrow 0$ of the global solutions to the double Riemann problem (1.1) and (1.6) are in agreement with those to the Riemann problem (1.1) and (1.7) for all the possible situations. As a consequence, it can be concluded that the solutions to the Riemann problem (1.1) and (1.7) are stable with respect to the specific small perturbation in the form (1.6) of the Riemann initial data (1.7).

The paper is organized in the following way. In Sect. 2, we study the Riemann problem (1.1) and (1.7) in detail. More precisely, the exact solutions of the Riemann problem (1.1) and (1.7) are obtained in fully explicit forms for all the possible Riemann initial data. In Sect. 3, the global solutions to the special initial value problem (1.1) and (1.6) are also constructed by studying all the occurring wave interactions. Then, the stabilities of Riemann solutions with respect to the small perturbations of the corresponding Riemann initial data can be obtained by taking the limit $x_{0} \rightarrow 0$ in the global solutions to the special initial value problem (1.1) and (1.6).

\section{The solutions of the Riemann problem (1.1) and (1.7)}

In this section, we are mainly concerned with the Riemann problem for system (1.1) subject to the Riemann initial data (1.7). We can refer to the literature $[1,8,9]$ about the Riemann problem for the symmetric Keyfitz-Kranzer system (1.4). In what follows, we first deliver some basic properties of system (1.1), which can also be seen in [5]. A simple calculation shows that the two eigenvalues of system (1.1) are given respectively by

$$
\lambda_{1}=\phi(r), \quad \lambda_{2}=\phi(r)+n r \phi^{\prime}(r) .
$$

It suffices to get $\lambda_{1}<\lambda_{2}$ when $r>0$ or $\lambda_{1}=\lambda_{2}$ when $r=0$ under the assumption $\phi^{\prime}(r)>0$. Hence system (1.1) is strictly hyperbolic in the quarter $(u, v)$ phase plane except for the ori- 
$\operatorname{gin}(u, v)=(0,0)$ where it is degenerated to be non-strictly hyperbolic. The corresponding right eigenvalues can be chosen as

$$
\overrightarrow{r_{1}}=\left(-v^{n-1}, u^{n-1}\right)^{T}, \quad \overrightarrow{r_{2}}=(u, v)^{T} .
$$

Let us introduce the notation $\nabla=\left(\frac{\partial}{\partial u}, \frac{\partial}{\partial \nu}\right)$, then we can get

$$
\nabla \lambda_{1} \cdot r_{1}=0, \quad \nabla \lambda_{2} \cdot r_{2}=n r\left((n+1) \phi^{\prime}(r)+n r \phi^{\prime \prime}(r)\right) \neq 0 \quad \text { when } r>0
$$

which implies that the characteristic field associated with $\lambda_{1}$ is always linearly degenerate and the characteristic field associated with $\lambda_{2}$ is genuinely nonlinear except for the origin. As a consequence, the elementary wave corresponding to $\lambda_{1}$ is always the contact discontinuity, and the elementary wave corresponding to $\lambda_{2}$ is either the shock wave or the rarefaction wave depending on the choice of the Riemann initial data (1.7).

Since the Riemann problem (1.1) and (1.7) remains unchanged under the uniform stretching of coordinates: $(x, t) \rightarrow(\alpha x, \alpha t)$, we can carry out the corresponding self-similar transformation

$$
(u, v)(x, t)=(u, v)(\xi), \quad \text { where } \xi=\frac{x}{t} .
$$

Under the self-similar transformation (2.4), the Riemann problem (1.1) and (1.7) can be reduced into the boundary value problem $[34,35]$ of ordinary differential equations as follows:

$$
\left\{\begin{array}{l}
\left(\phi(r)-\xi+n u^{n} \phi^{\prime}(r)\right) u_{\xi}+n u v^{n-1} \phi^{\prime}(r) v_{\xi}=0 \\
n u^{n-1} v \phi^{\prime}(r) u_{\xi}+\left(\phi(r)-\xi+n v^{n} \phi^{\prime}(r)\right) v_{\xi}=0 \\
(u, v)( \pm \infty)=\left(u_{ \pm}, v_{ \pm}\right)
\end{array}\right.
$$

Furthermore, (2.5) can be rewritten as

$$
\left(\begin{array}{cc}
\phi(r)-\xi+n u^{n} \phi^{\prime}(r) & n u v^{n-1} \phi^{\prime}(r) \\
n u^{n-1} v \phi^{\prime}(r) & \phi(r)-\xi+n v^{n} \phi^{\prime}(r)
\end{array}\right)\left(\begin{array}{l}
d u \\
d v
\end{array}\right)=\left(\begin{array}{l}
0 \\
0
\end{array}\right) .
$$

It is easy to know that (2.6) provides either the constant state solution or the singular solution, namely the contact discontinuity corresponds to $\lambda_{1}$ and the rarefaction wave corresponds to $\lambda_{2}$. For a given left state $\left(u_{-}, v_{-}\right)$in the interior of the quarter $(u, v)$ phase plane, on the one hand, the contact discontinuity is expressed in the quarter $(u, v)$ phase plane as follows:

$$
J: \tau=\phi(r)=\phi\left(r_{-}\right), \quad \text { where } r=u^{n}+v^{n} \text { and } r_{-}=u_{-}^{n}+v_{-}^{n} .
$$

It is easily seen from (2.7) that we have $u^{n}+v^{n}=u_{-}^{n}+v_{-}^{n}$ under the assumption $\phi^{\prime}(r)>0$. By differentiating $u$ with respect to $v$ in the relation formula $u^{n}+v^{n}=u_{-}^{n}+v_{-}^{n}$, we get

$$
\frac{d u}{d v}=-\frac{v^{n-1}}{u^{n-1}}, \quad \frac{d^{2} u}{d^{2} v}=-\frac{(n-1) v^{n-2}}{u^{n-1}} .
$$


It can be deduced directly from (2.8) that we have $\frac{d u}{d v}<0$ and $\frac{d^{2} u}{d^{2} v}<0$ when $n>1$. On the other hand, the rarefaction wave is expressed in the quarter $(u, v)$ phase plane as follows:

$$
R: \xi=\phi(r)+n \phi^{\prime}(r) \quad \text { when } \frac{u}{v}=\frac{u_{-}}{v_{-}} \text {and } r>r_{-} .
$$

Let us turn our attention to the discontinuous solutions of system (1.1). For a bounded discontinuity on $\xi=\sigma$, there exist the following Rankine-Hugoniot jump conditions:

$$
\left\{\begin{array}{l}
\sigma[u]=[u \phi(r)], \\
\sigma[v]=[v \phi(r)],
\end{array}\right.
$$

where $\sigma=\frac{d x}{d t}$. The jump quantity $[r]$ is calculated by $[r]=r-r_{-}$, in which $r_{-}($or $r$ ) is the value of $r$ on the left-hand (or right-hand) side of the discontinuity. For a given left state $\left(u_{-}, v_{-}\right)$in the interior of the quarter $(u, v)$ phase plane, the contact discontinuity is also expressed by $(2.7)$ and the shock wave is expressed in the quarter $(u, v)$ phase plane as follows:

$$
S: \sigma=\frac{u \phi(r)-u_{-} \phi\left(r_{-}\right)}{u-u_{-}} \quad \text { when } \frac{u}{v}=\frac{u_{-}}{v_{-}} \text {and } r<r_{-} .
$$

It can be seen from (2.9) and (2.11) that the rarefaction wave curve has the same expression formula as the shock wave curve. Thus, system (1.1) is attributed to the well-known Temple class [6, 7].

In the end, we use the above elementary waves $J, R$, and $S$ to construct the solutions of the Riemann problem (1.1) and (1.7) in the quarter $(u, v)$ phase plane by using the method of phase plane analysis. For the given left state $\left(u_{-}, v_{-}\right)$in the interior of the quarter $(u, v)$ phase plane, the solutions of the Riemann problem (1.1) and (1.7) are a contact discontinuity when $r_{-}=r_{+}$, or a rarefaction wave when $\frac{u_{+}}{v_{+}}=\frac{u_{-}}{v_{-}}$and $r_{+}>r_{-}$, or a shock wave when $\frac{u_{+}}{v_{+}}=\frac{u_{-}}{v_{-}}$and $r_{+}<r_{-}$to connect with the two constant states $\left(u_{ \pm}, v_{ \pm}\right)$directly. For other general situations, the solutions of the Riemann problem (1.1) and (1.7) have the following two kinds of combinations of elementary waves according to $0<r_{-}<r_{+}$or not.

(1) If $0<r_{+}<r_{-}$, then the solutions of the Riemann problem (1.1) and (1.7) can be expressed by the symbol $J_{1}+S_{2}$ in the following form:

$$
(u, v)(x, t)= \begin{cases}\left(u_{-}, v_{-}\right), & x<\tau_{1} t, \\ \left(u_{*}, v_{*}\right), & \tau_{1} t<x<\sigma_{2} t, \\ \left(u_{+}, v_{+}\right), & x>\sigma_{2} t,\end{cases}
$$

in which the intermediate state $\left(u_{*}, v_{*}\right)$ between $J_{1}$ and $S_{2}$ is calculated by

$$
\left(u_{*}, v_{*}\right)=\left(\sqrt[n]{\frac{u_{-}^{n}+v_{-}^{n}}{u_{+}^{n}+v_{+}^{n}}} u_{+}, \sqrt[n]{\frac{u_{-}^{n}+v_{-}^{n}}{u_{+}^{n}+v_{+}^{n}}} v_{+}\right) .
$$


Here $\tau_{1}=\phi\left(r_{-}\right)$is the propagation speed of the contact discontinuity $J_{1}$ and $\sigma_{2}$ is used to denote the propagation speed of the shock wave $S_{2}$, which can be calculated by

$$
\sigma_{2}=\frac{u_{+} \phi\left(r_{+}\right)-u_{*} \phi\left(r_{*}\right)}{u_{+}-u_{*}}=\frac{u_{+} \phi\left(r_{+}\right)-\sqrt[n]{\frac{u_{-}^{n}+v_{n}^{n}}{u_{+}^{n}+v_{+}^{n}}} u_{+} \phi\left(r_{-}\right)}{u_{+}-\sqrt[n]{\frac{u_{-}^{n}+v_{n}^{n}}{u_{+}^{n}+v_{+}^{n}}} u_{+}}=\frac{\sqrt[n]{r_{+}} \phi\left(r_{+}\right)-\sqrt[n]{r_{-}} \phi\left(r_{-}\right)}{\sqrt[n]{r_{+}}-\sqrt[n]{r_{-}}} .
$$

The symbol $J_{1}+S_{2}$ is used to represent the 1-contact discontinuity $J_{1}$ followed by the 2shock wave $S_{2}$ in this paper and the similar symbol $J_{1}+R_{2}$ will also be used later without explanation again.

(2) If $0<r_{-}<r_{+}$, then the solution of the Riemann problem (1.1) and (1.7) can be expressed by the symbol $J_{1}+R_{2}$ in the following form:

$$
(u, v)(x, t)= \begin{cases}\left(u_{-}, v_{-}\right), & x<\tau_{1} t, \\ \left(u_{*}, v_{*}\right), & \tau_{1} t<x<\lambda_{2}\left(r_{-}\right) t, \\ (u, v), & \lambda_{2}\left(r_{-}\right) t \leq x \leq \lambda_{2}\left(r_{+}\right) t \\ \left(u_{+}, v_{+}\right), & x>\lambda_{2}\left(r_{+}\right) t\end{cases}
$$

in which $\tau_{1}$ and $\left(u_{*}, v_{*}\right)$ can be calculated in the same way as before. The state $(u, v)$ in the rarefaction wave fan $R_{2}$ varies from $\left(u_{*}, v_{*}\right)$ to $\left(u_{+}, v_{+}\right)$, which can be determined uniquely by

$$
\frac{u}{v}=\frac{u_{+}}{v_{+}}, \quad \frac{x}{t}=\phi(r)+n r \phi^{\prime}(r) .
$$

In addition, the propagation speeds of characteristics in the rarefaction wave fan $R_{2}$ can be calculated by $\xi=\lambda_{2}(r)=\phi(r)+n r \phi^{\prime}(r)$, in which $r$ varies from $r_{*}\left(=r_{-}\right)$to $r_{+}$.

\section{Construction of global solutions to the double Riemann problem (1.1) and}

\section{(1.6)}

In this section, the main purpose is to construct the global solutions to the double Riemann problem (1.1) and (1.6) by using the method of characteristics. In the present work, we restrict ourselves to considering the initial data (1.6) which are located in the quarter $(u, v)$ phase plane. In order to cover all the cases completely, we divide our discussion into the following four cases according to the different combinations of the Riemann solutions starting from the initial points $(0,0)$ and $\left(x_{0}, 0\right)$ as follows:
(1) $J+S$ and $J+S$,
(2) $J+R$ and $J+R$,
(3) $J+S$ and $J+R$,
(4) $J+R$ and $J+S$.

Furthermore, we make a step further to consider the interesting work of determining whether the limits $x_{0} \rightarrow 0$ of the solutions to the double Riemann problem (1.1) and (1.6) are in accordance with the corresponding ones to the Riemann problem (1.1) and (1.7).

Case $1 J+S$ and $J+S$. 


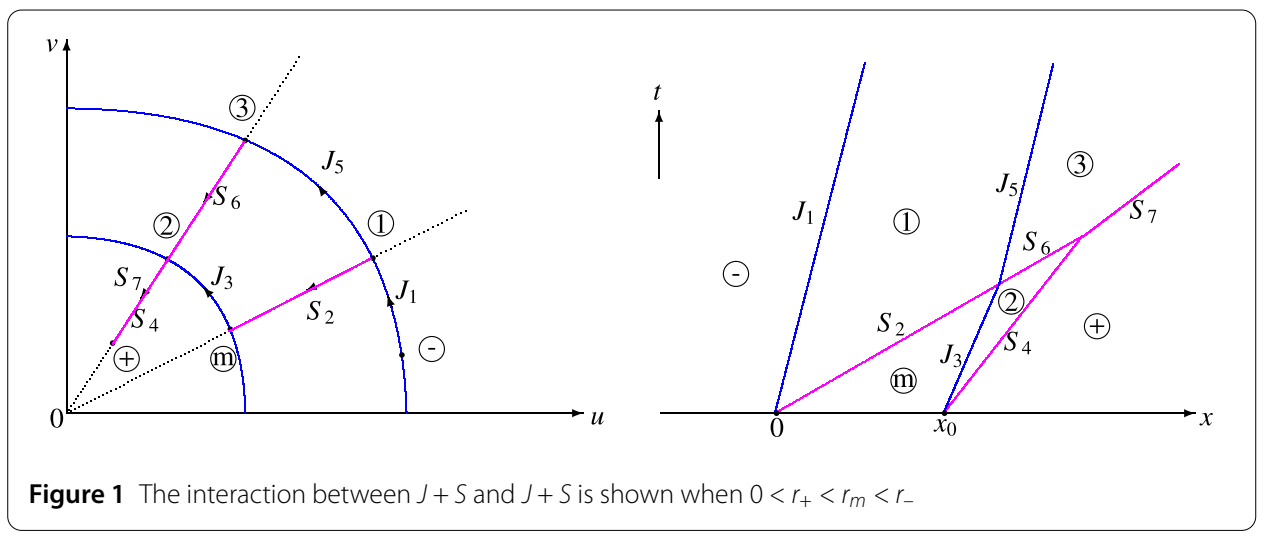

We first consider the situation that there is a contact discontinuity followed by a shock wave emitting from the initial points $(0,0)$ and $\left(x_{0}, 0\right)$, respectively. Obviously, the occurrence of this case depends on the conditions $0<r_{+}<r_{m}<r_{-}$. For convenience, we use $J_{1}$, $S_{2}$ and $J_{3}, S_{4}$ to denote the contact discontinuities and the shock waves emitting from the initial points $(0,0)$ and $\left(x_{0}, 0\right)$ (see Fig. 1$)$. In this case, when the time $t$ is small enough, the solution to the double Riemann problem (1.1) and (1.6) can be expressed briefly as (see Fig. 1):

$$
\left(u_{-}, v_{-}\right)+J_{1}+\left(u_{1}, v_{1}\right)+S_{2}+\left(u_{m}, v_{m}\right)+J_{3}+\left(u_{2}, v_{2}\right)+S_{4}+\left(u_{+}, v_{+}\right)
$$

where the symbol " + " means "followed by". It follows from (2.13) that the intermediate states $\left(u_{1}, v_{1}\right)$ and $\left(u_{2}, v_{2}\right)$ may be given respectively by

$$
\begin{aligned}
& \left(u_{1}, v_{1}\right)=\left(\sqrt[n]{\frac{u_{-}^{n}+v_{-}^{n}}{u_{m}^{n}+v_{m}^{n}}} u_{m}, \sqrt[n]{\frac{u_{-}^{n}+v_{-}^{n}}{u_{m}^{n}+v_{m}^{n}}} v_{m}\right), \\
& \left(u_{2}, v_{2}\right)=\left(\sqrt[n]{\frac{u_{m}^{n}+v_{m}^{n}}{u_{+}^{n}+v_{+}^{n}}} u_{+}, \sqrt[n]{\frac{u_{m}^{n}+v_{m}^{n}}{u_{+}^{n}+v_{+}^{n}}} v_{+}\right) .
\end{aligned}
$$

It can be seen from Fig. 1 that the relations $r_{+}<r_{2}=r_{m}<r_{1}=r_{-}$can be established directly.

Lemma 3.1 The shock wave $S_{2}$ collides with the contact discontinuity $J_{3}$ in finite time. Then the interaction between $S_{2}$ and $J_{3}$ gives rise to a new contact discontinuity and a new shock wave denoted by $J_{5}$ and $S_{6}$, respectively. More precisely, the two contact discontinuities $J_{1}$ and $J_{5}$ are parallel to each other, while the two shock waves $S_{2}$ and $S_{6}$ share the same propagation speed.

Proof It is easy to know that the propagation speeds of $S_{2}$ and $J_{3}$ are given respectively by

$$
\sigma_{2}=\frac{\sqrt[n]{r_{m}} \phi\left(r_{m}\right)-\sqrt[n]{r_{-}} \phi\left(r_{-}\right)}{\sqrt[n]{r_{m}}-\sqrt[n]{r_{-}}}, \quad \tau_{3}=\phi\left(r_{m}\right)
$$

By virtue of $r_{m}<r_{-}$and the assumption $\phi^{\prime}(r)>0$, we have

$$
\sigma_{2}-\tau_{3}=\frac{\sqrt[n]{r_{-}}\left(\phi\left(r_{m}\right)-\phi\left(r_{-}\right)\right)}{\sqrt[n]{r_{m}}-\sqrt[n]{r_{-}}}>0
$$


which implies that $\sigma_{2}>\tau_{3}$. In other words, the shock wave $S_{2}$ collides with the contact discontinuity $J_{3}$ in finite time. The intersection point $\left(x_{1}, t_{1}\right)$ is determined by

$$
\left\{\begin{array}{l}
x_{1}=\sigma_{2} t_{1}=\frac{\sqrt[n]{r_{m}} \phi\left(r_{m}\right)-\sqrt[n]{r_{-}} \phi\left(r_{-}\right)}{\sqrt[n]{r_{m}}-\sqrt[n]{r_{-}}} t_{1} \\
x_{1}-x_{0}=\tau_{3} t_{1}=\phi\left(r_{m}\right) t_{1}
\end{array}\right.
$$

which yields

$$
\left(x_{1}, t_{1}\right)=\left(\frac{x_{0}\left(\sqrt[n]{r_{m}} \phi\left(r_{m}\right)-\sqrt[n]{r_{-}} \phi\left(r_{-}\right)\right)}{\sqrt[n]{r_{-}}\left(\phi\left(r_{m}\right)-\phi\left(r_{-}\right)\right)}, \frac{x_{0}\left(\sqrt[n]{r_{m}}-\sqrt[n]{r_{-}}\right)}{\sqrt[n]{r_{-}}\left(\phi\left(r_{m}\right)-\phi\left(r_{-}\right)\right)}\right)
$$

The collision between the shock wave $S_{2}$ and the contact discontinuity $J_{3}$ occurs at the point $\left(x_{1}, t_{1}\right)$, where we again have a local Riemann problem for system (1.1) subject to the Riemann-type initial data $\left(u_{1}, v_{1}\right)$ and $\left(u_{2}, v_{2}\right)$. Due to the relations $r_{2}=r_{m}<r_{-}=r_{1}$, the solution to the local Riemann problem at the point $\left(x_{1}, t_{1}\right)$ is still a contact discontinuity followed by a shock wave, which are denoted by $J_{5}$ and $S_{6}$, respectively. In addition, the intermediate state $\left(u_{3}, v_{3}\right)$ between $J_{5}$ and $S_{6}$ can be obtained by

$$
\left(u_{3}, v_{3}\right)=\left(\sqrt[n]{\frac{u_{-}^{n}+v_{-}^{n}}{u_{+}^{n}+v_{+}^{n}}} u_{+}, \sqrt[n]{\frac{u_{-}^{n}+v_{-}^{n}}{u_{+}^{n}+v_{+}^{n}}} v_{+}\right)
$$

The propagation speeds of $J_{5}$ and $S_{6}$ can be computed by $\tau_{5}=\phi\left(r_{1}\right)=\phi\left(r_{-}\right)$and $\sigma_{6}=$ $\frac{\sqrt[n]{r_{2}} \phi\left(r_{2}\right)-\sqrt[n]{r_{3}} \phi\left(r_{3}\right)}{\sqrt[n]{r_{2}}-\sqrt[n]{r_{3}}}=\frac{\sqrt[n]{r_{m}} \phi\left(r_{m}\right)-\sqrt[n]{r_{-}} \phi\left(r_{-}\right)}{\sqrt[n]{r_{m}}-\sqrt[n]{r_{-}}}$, respectively. On the one hand, it is worth mentioning that the propagation speed of $J_{1}$ is also calculated by $\tau_{1}=\phi\left(r_{-}\right)$, which implies that $J_{1}$ is parallel to $J_{5}$. On the other hand, we can also obtain $\sigma_{2}=\sigma_{6}$, which means that the shock wave keeps its propagation direction unchanged when it passes through the contact discontinuity.

In the following, we consider the confluence of the two shock waves $S_{6}$ and $S_{4}$.

Lemma 3.2 The two shock waves $S_{4}$ and $S_{6}$ coalesce into a new shock wave denoted by $S_{7}$.

Proof The propagation speed of $S_{4}$ is given by $\sigma_{4}=\frac{\sqrt[n]{r_{+}} \phi\left(r_{+}\right)-\sqrt[n]{r_{2}} \phi\left(r_{2}\right)}{\sqrt[n]{r_{+}}-\sqrt[n]{r_{2}}}=\frac{\sqrt[n]{r_{+}} \phi\left(r_{+}\right)-\sqrt[n]{r_{m}} \phi\left(r_{m}\right)}{\sqrt[n]{r_{+}}-\sqrt[n]{r_{m}}}$. Comparing with $\sigma_{6}$, we have

$$
\begin{aligned}
\sigma_{6} & -\sigma_{4} \\
& =\frac{\sqrt[n]{r_{m}} \phi\left(r_{m}\right)-\sqrt[n]{r_{-}} \phi\left(r_{-}\right)}{\sqrt[n]{r_{m}}-\sqrt[n]{r_{-}}}-\frac{\sqrt[n]{r_{+}} \phi\left(r_{+}\right)-\sqrt[n]{r_{m}} \phi\left(r_{m}\right)}{\sqrt[n]{r_{+}}-\sqrt[n]{r_{m}}} \\
& =\frac{\left(\sqrt[n]{r_{+}}-\sqrt[n]{r_{m}}\right)\left(\sqrt[n]{r_{m}} \phi\left(r_{m}\right)-\sqrt[n]{r_{-}} \phi\left(r_{-}\right)\right)-\left(\sqrt[n]{r_{m}}-\sqrt[n]{r_{-}}\right)\left(\sqrt[n]{r_{+}} \phi\left(r_{+}\right)-\sqrt[n]{r_{m}} \phi\left(r_{m}\right)\right)}{\left(\sqrt[n]{r_{m}}-\sqrt[n]{r_{-}}\right)\left(\sqrt[n]{r_{+}}-\sqrt[n]{r_{m}}\right)} \\
& =\frac{\sqrt[n]{r_{m} r_{+}}\left(\phi\left(r_{m}\right)-\phi\left(r_{+}\right)\right)+\sqrt[n]{r_{+} r_{-}}\left(\phi\left(r_{+}\right)-\phi\left(r_{-}\right)\right)+\sqrt[n]{r_{-} r_{m}}\left(\phi\left(r_{-}\right)-\phi\left(r_{m}\right)\right)}{\left(\sqrt[n]{r_{m}}-\sqrt[n]{r_{-}}\right)\left(\sqrt[n]{r_{+}}-\sqrt[n]{r_{m}}\right)} \\
& =\frac{\left(\sqrt[n]{r_{m} r_{+}}-\sqrt[n]{r_{+} r_{-}}\right)\left(\phi\left(r_{m}\right)-\phi\left(r_{+}\right)\right)+\left(\sqrt[n]{r_{m} r_{-}}-\sqrt[n]{r_{+} r_{-}}\right)\left(\phi\left(r_{-}\right)-\phi\left(r_{m}\right)\right)}{\left(\sqrt[n]{r_{m}}-\sqrt[n]{r_{-}}\right)\left(\sqrt[n]{r_{+}}-\sqrt[n]{r_{m}}\right)} \\
& =\sqrt[n]{r_{-}} \frac{\phi\left(r_{-}\right)-\phi\left(r_{m}\right)}{\sqrt[n]{r_{-}}-\sqrt[n]{r_{m}}}-\sqrt[n]{r_{+}} \frac{\phi\left(r_{m}\right)-\phi\left(r_{+}\right)}{\sqrt[n]{r_{m}}-\sqrt[n]{r_{+}}}
\end{aligned}
$$


By using the mean value theorem, there exist $\overline{r_{1}}$ and $\overline{r_{2}}$ satisfying $r_{+}<\overline{r_{2}}<r_{m}<\overline{r_{1}}<r_{-}$such that we have

$$
\sigma_{6}-\sigma_{4}=n \bar{r}_{1} \frac{n-1}{n} \phi^{\prime}\left(\overline{r_{1}}\right) \sqrt[n]{r_{-}}-n \overline{r_{2}} \frac{n-1}{n} \phi^{\prime}\left(\overline{r_{2}}\right) \sqrt[n]{r_{+}},
$$

in which we have used the formula $\frac{\partial \phi}{\partial \sqrt[n]{r}}=\frac{\partial \phi}{\partial r} \frac{\partial r}{\partial \sqrt[n]{r}}=n r^{\frac{n-1}{n}} \phi^{\prime}(r)$. Under the assumption $\phi^{\prime \prime}(r)>0$, we can take a step further to get $\phi^{\prime}\left(\overline{r_{1}}\right)>\phi^{\prime}\left(\overline{r_{2}}\right)$ when $\overline{r_{1}}>\overline{r_{2}}$. Noticing that $n>1$, we also have $\overline{r_{1}} \frac{n-1}{n}>\overline{r_{2}} \frac{n-1}{n}$ when $\overline{r_{1}}>\overline{r_{2}}$. Hence, we have $\sigma_{6}>\sigma_{4}$. In other words, the shock wave $S_{6}$ catches up with $S_{4}$ in finite time.

The intersection point $\left(x_{2}, t_{2}\right)$ is determined by

$$
\left\{\begin{array}{l}
x_{2}=\sigma_{6} t_{2}=\frac{\sqrt[n]{r_{m}} \phi\left(r_{m}\right)-\sqrt[n]{r_{-}} \phi\left(r_{-}\right)}{\sqrt[n]{r_{m}}-\sqrt[n]{r_{-}}} t_{2} \\
x_{2}-x_{0}=\sigma_{4} t_{2}=\frac{\sqrt[n]{r_{+}} \phi\left(r_{+}\right)-\sqrt[n]{r_{m}} \phi\left(r_{m}\right)}{\sqrt[n]{r_{+}}-\sqrt[n]{r_{m}}} t_{2}
\end{array}\right.
$$

which yields

$$
\left\{\begin{array}{l}
x_{2}=\frac{x_{0}\left(\sqrt[n]{r_{m}}-\sqrt[n]{r_{+}}\right)\left(\sqrt[n]{r_{-}} \phi\left(r_{-}\right)-\sqrt[n]{r_{m}} \phi\left(r_{m}\right)\right)}{\left.\sqrt[n]{r_{+}}\left(\sqrt[n]{r_{m}}-\sqrt[n]{r_{-}}\right)\left(\phi\left(r_{m}\right)-\phi\left(r_{+}\right)\right)+\sqrt[n]{r_{-}}-\sqrt[n]{r_{m}}-\sqrt[n]{r_{+}}\right)\left(\phi\left(r_{-}\right)-\phi\left(r_{m}\right)\right)}, \\
t_{2}=\frac{x_{0}\left(\sqrt[n]{r_{m}}-\sqrt[n]{r_{-}}\right)\left(\sqrt[n]{r_{-}}-\sqrt[n]{r_{m}}\right)}{\sqrt[n]{r_{+}}\left(\sqrt[n]{r_{m}}-\sqrt[n]{r_{-}}\right)\left(\phi\left(r_{m}\right)-\phi\left(r_{+}\right)\right)+\sqrt[n]{r_{-}}\left(\sqrt[n]{r_{m}}-\sqrt[n]{r_{+}}\right)\left(\phi\left(r_{-}\right)-\phi\left(r_{m}\right)\right)}
\end{array}\right.
$$

It can be deduced from (3.8) that $\frac{u_{3}}{v_{3}}=\frac{u_{+}}{v_{+}}$. In addition, one also has $r_{+}<r_{-}=r_{3}$. Thus, the conclusion can be drawn that the two states $\left(u_{3}, v_{3}\right)$ and $\left(u_{+}, v_{+}\right)$are connected directly by a new shock wave $S_{7}$, which can also be seen from the $(u, v)$ phase plane in Fig. 1 . It means that the two shock waves $S_{4}$ and $S_{6}$ coalesce into a new shock wave which is denoted by $S_{7}$, whose propagation speed is given by $\sigma_{7}=\frac{\sqrt[n]{r_{+}} \phi\left(r_{+}\right)-\sqrt[n]{r_{-}} \phi\left(r_{-}\right)}{\sqrt[n]{r_{+}}-\sqrt[n]{r_{-}}}$. On the one hand, we have

$$
\begin{aligned}
\sigma_{6}-\sigma_{7} & =\frac{\sqrt[n]{r_{m}} \phi\left(r_{m}\right)-\sqrt[n]{r_{-}} \phi\left(r_{-}\right)}{\sqrt[n]{r_{m}}-\sqrt[n]{r_{-}}}-\frac{\sqrt[n]{r_{+}} \phi\left(r_{+}\right)-\sqrt[n]{r_{-}} \phi\left(r_{-}\right)}{\sqrt[n]{r_{+}}-\sqrt[n]{r_{-}}} \\
& =\sqrt[n]{r_{m}} \frac{\phi\left(r_{-}\right)-\phi\left(r_{m}\right)}{\sqrt[n]{r_{-}}-\sqrt[n]{r_{m}}}-\sqrt[n]{r_{+}} \frac{\phi\left(r_{-}\right)-\phi\left(r_{+}\right)}{\sqrt[n]{r_{-}}-\sqrt[n]{r_{+}}}
\end{aligned}
$$

On the other hand, we also have

$$
\begin{aligned}
\sigma_{4}-\sigma_{7} & =\frac{\sqrt[n]{r_{+}} \phi\left(r_{+}\right)-\sqrt[n]{r_{m}} \phi\left(r_{m}\right)}{\sqrt[n]{r_{+}}-\sqrt[n]{r_{m}}}-\frac{\sqrt[n]{r_{+}} \phi\left(r_{+}\right)-\sqrt[n]{r_{-}} \phi\left(r_{-}\right)}{\sqrt[n]{r_{+}}-\sqrt[n]{r_{-}}} \\
& =\sqrt[n]{r_{m}} \frac{\phi\left(r_{m}\right)-\phi\left(r_{+}\right)}{\sqrt[n]{r_{m}}-\sqrt[n]{r_{+}}}-\sqrt[n]{r_{-}} \frac{\phi\left(r_{-}\right)-\phi\left(r_{+}\right)}{\sqrt[n]{r_{-}}-\sqrt[n]{r_{+}}} .
\end{aligned}
$$

With the similar calculation as that in Lemma 3.2, the following inequalities $\sigma_{6}-\sigma_{7}>0$ and $\sigma_{4}-\sigma_{7}<0$ can also be established, which means that the propagation speed of the shock wave $S_{7}$ is between those of $S_{4}$ and $S_{6}$.

Case $2 J+R$ and $J+R$.

We consider the situation that there is a contact discontinuity followed by a rarefaction wave emitting from the initial points $(0,0)$ and $\left(x_{0}, 0\right)$, respectively. The occurrence of this case depends on the condition $0<r_{-}<r_{m}<r_{+}$. We use $J_{1}, R_{2}$ and $J_{3}, R_{4}$ to denote 

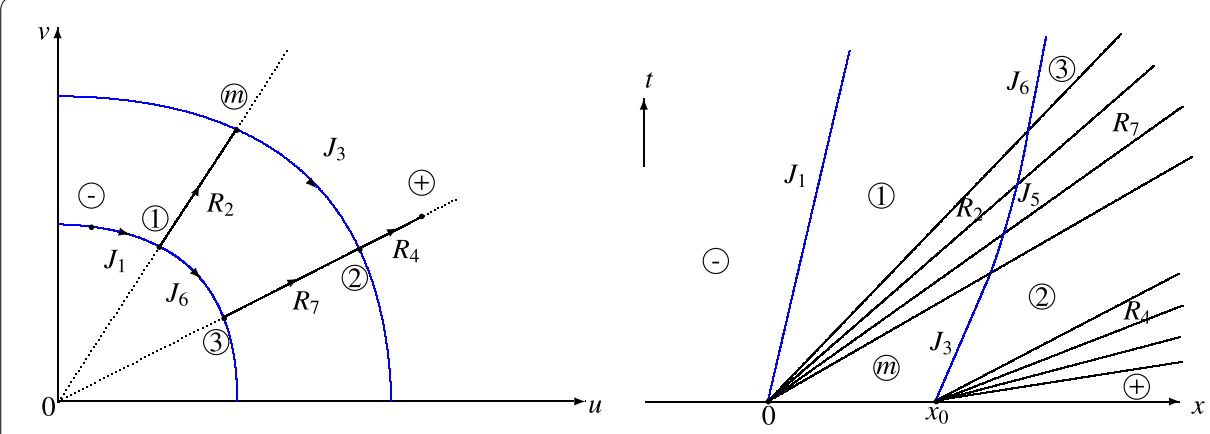

Figure 2 The interaction between $J+R$ and $J+R$ is shown when $0<r_{-}<r_{m}<r_{+}$

the contact discontinuities and the shock waves emitting from the initial points $(0,0)$ and $\left(x_{0}, 0\right)$ (see Fig. 2). In this case, when the time $t$ is small enough, the solution to the double Riemann problem (1.1) and (1.6) may be represented succinctly as follows (see Fig. 2):

$$
\left(u_{-}, v_{-}\right)+J_{1}+\left(u_{1}, v_{1}\right)+R_{2}+\left(u_{m}, v_{m}\right)+J_{3}+\left(u_{2}, v_{2}\right)+R_{4}+\left(u_{+}, v_{+}\right) .
$$

The intermediate states $\left(u_{1}, v_{1}\right),\left(u_{2}, v_{2}\right)$, and $\left(u_{3}, v_{3}\right)$ are the same as in Case 1 . Furthermore, it can be seen from Fig. 2 that the relations $r_{1}=r_{-}<r_{2}=r_{m}<r_{+}$can be established directly.

Lemma 3.3 The wave front of the rarefaction wave $R_{2}$ collides with the contact discontinuity $J_{3}$ in finite time. Then the interaction between $R_{2}$ and $J_{3}$ gives rise to a new contact discontinuity and a new rarefaction wave denoted by $J_{6}$ and $R_{7}$, respectively. More precisely, the two contact discontinuities $J_{1}$ and $J_{6}$ are parallel to each other, while the wave front of the rarefaction wave $R_{7}$ and the wave back of the rarefaction wave $R_{4}$ share the same propagation speed.

Proof A simple calculation shows that the propagation speeds of the wave front of the rarefaction wave $R_{2}$ and the contact discontinuity $J_{3}$ are given respectively by

$$
\xi_{2}\left(u_{m}, v_{m}\right)=\phi\left(r_{m}\right)+n r_{m} \phi^{\prime}\left(r_{m}\right), \quad \tau_{3}=\phi\left(r_{m}\right) .
$$

We have $\xi_{2}\left(u_{m}, v_{m}\right)-\tau_{3}=n r_{m} \phi^{\prime}\left(r_{m}\right)>0$ under the assumption $\phi^{\prime}(r)>0$, which implies that $\xi_{2}\left(u_{m}, v_{m}\right)>\tau_{3}$. In other words, the rarefaction wave $R_{2}$ collides with the contact discontinuity $J_{3}$ in finite time. The intersection point $\left(x_{1}, t_{1}\right)$ is determined by

$$
\left\{\begin{array}{l}
x_{1}=\xi_{2}\left(u_{m}, v_{m}\right) t_{1}=\left(\phi\left(r_{m}\right)+n r_{m} \phi^{\prime}\left(r_{m}\right)\right) t_{1}, \\
x_{1}-x_{0}=\tau_{3} t_{1}=\phi\left(r_{m}\right) t_{1},
\end{array}\right.
$$

from which it is easy to get

$$
\left(x_{1}, t_{1}\right)=\left(\frac{x_{0}\left(\phi\left(r_{m}\right)+n r_{m} \phi^{\prime}\left(r_{m}\right)\right)}{n r_{m} \phi^{\prime}\left(r_{m}\right)}, \frac{x_{0}}{n r_{m} \phi^{\prime}\left(r_{m}\right)}\right) .
$$

The collision between the wave front of the rarefaction wave $R_{2}$ and the contact discontinuity $J_{3}$ occurs at the point $\left(x_{1}, t_{1}\right)$. The contact discontinuity $J_{3}$ begins to penetrate the 
rarefaction wave $R_{2}$ after $t_{1}$, which is denoted by $J_{5}$ during the process of penetration. The expression for $J_{5}$ during the process of penetration is determined by

$$
\left\{\begin{array}{l}
\frac{d x}{d t}=\phi(r), \\
\frac{x}{t}=\phi(r)+n r \phi^{\prime}(r), \\
x\left(t_{1}\right)=x_{1},
\end{array}\right.
$$

in which $r$ changes from $r_{m}$ to $r_{1}\left(=r_{-}\right)$. It can be deduced from the second equation in (3.19) that

$$
\frac{d x}{d t}=\phi(r)+n r \phi^{\prime}(r)+\left((n+1) \phi^{\prime}(r)+n r \phi^{\prime \prime}(r)\right) t \cdot \frac{d r}{d t},
$$

which, together with the first equation in (3.19), yields

$$
\frac{d t}{t}=-\frac{\left((n+1) \phi^{\prime}(r)+n r \phi^{\prime \prime}(r)\right) d r}{n r \phi^{\prime}(r)},
$$

which enables us to have

$$
d \ln t=-\frac{d \ln r}{n}-d \ln \left(n r \phi^{\prime}(r)\right)=-d \ln \left(n r^{\frac{1+n}{n}} \phi^{\prime}(r)\right) .
$$

With the initial condition $r\left(t_{1}\right)=r_{m}$ in mind, one can only obtain the following implicit expression:

$$
r^{\frac{1+n}{n}} \phi^{\prime}(r) t=\left(r_{m}\right)^{\frac{1+n}{n}} \phi^{\prime}\left(r_{m}\right) t_{1}=\frac{\left(r_{m}\right)^{\frac{1}{n}} x_{0}}{n} .
$$

In addition, it follows from (3.21) that

$$
\frac{d r}{d t}=\frac{-n r \phi^{\prime}(r)}{\left((n+1) \phi^{\prime}(r)+n r \phi^{\prime \prime}(r)\right) t} .
$$

In the same way as before, by differentiating the first equation in (3.19) with respect to $t$, we have

$$
\frac{d^{2} x}{d t^{2}}=\phi^{\prime}(r) \frac{d r}{d t}=\frac{-n r\left(\phi^{\prime}(r)\right)^{2}}{\left((n+1) \phi^{\prime}(r)+n r \phi^{\prime \prime}(r)\right) t}<0,
$$

in which (3.24) and the assumptions $\phi^{\prime}>0$ and $\phi^{\prime \prime}>0$ have been used, which implies that the contact discontinuity slows down during the process of penetration.

Due to the facts $r_{2}=r_{m}$ and $r_{1}=r_{3}=r_{-}$, it is worthwhile to notice that

$$
\begin{aligned}
& \phi\left(r_{m}\right)+n r_{m} \phi^{\prime}\left(r_{m}\right)=\phi\left(r_{2}\right)+n r_{2} \phi^{\prime}\left(r_{2}\right) \quad \text { and } \\
& \phi\left(r_{1}\right)+n r_{1} \phi^{\prime}\left(r_{1}\right)=\phi\left(r_{3}\right)+n r_{3} \phi^{\prime}\left(r_{3}\right) .
\end{aligned}
$$

Thus, it can be seen clearly that the rarefaction wave does not change the propagation direction during the process of penetration. But here it should be stressed that the state in the rarefaction wave fan $R_{2}$ varying from $\left(u_{m}, v_{m}\right)$ to $\left(u_{1}, v_{1}\right)$ is determined by $\frac{u}{v}=\frac{u_{m}}{v_{m}}$ as 
well as the state in the rarefaction wave fan $R_{7}$ varying from $\left(u_{2}, v_{2}\right)$ to $\left(u_{3}, v_{3}\right)$ is governed by $\frac{u}{v}=\frac{u_{+}}{v_{+}}$.

It is clear to see that $\left(u_{3}, v_{3}\right)$ can be directly connected to $\left(u_{1}, v_{1}\right)$ through a contact discontinuity $J_{6}$ for the reason that $r_{1}=r_{3}=r_{-}$. Thus, $J_{5}$ is able to penetrate $R_{2}$ completely and ends at the point $\left(x_{2}, t_{2}\right)$, which is determined by

$$
\left\{\begin{array}{l}
x_{2}=\xi_{2}\left(u_{-}, v_{-}\right) t_{2}=\left(\phi\left(r_{-}\right)+n r_{-} \phi^{\prime}\left(r_{-}\right)\right) t_{2}, \\
x_{2}=\left(\phi\left(\left(\frac{n r_{m}^{-\frac{1}{n}} \phi^{\prime}(r) t}{x_{0}}\right)^{-\frac{n}{n+1}}\right)+n\left(\frac{n r_{m}^{-\frac{1}{n}} \phi^{\prime}(r) t}{x_{0}}\right)^{-\frac{n}{n+1}} \phi^{\prime}\left(\left(\frac{n r_{m}^{-\frac{1}{n}} \phi^{\prime}(r) t}{x_{0}}\right)^{-\frac{n}{n+1}}\right)\right) t,
\end{array}\right.
$$

which enables us to get

$$
\left\{\begin{array}{l}
x_{2}=\left(\phi\left(\left(\frac{n r_{m}^{-\frac{1}{n}} \phi^{\prime}(r) t}{x_{0}}\right)^{-\frac{n}{n+1}}\right)+n\left(\frac{n r_{m}^{-\frac{1}{n}} \phi^{\prime}(r) t}{x_{0}}\right)^{-\frac{n}{n+1}} \phi^{\prime}\left(\left(\frac{n r_{m}^{-\frac{1}{n}} \phi^{\prime}(r) t}{x_{0}}\right)^{-\frac{n}{n+1}}\right)\right) t, \\
t_{2}=\frac{\left(\phi\left(\left(\frac{n r_{m}^{-\frac{1}{n}} \phi^{\prime}(r) t}{x_{0}}\right)^{-\frac{n}{n+1}}\right)+n\left(\frac{n r_{m}^{-\frac{1}{n}} \phi^{\prime}(r) t}{x_{0}}\right)^{-\frac{n}{n+1}} \phi^{\prime}\left(\left(\frac{n r_{m}^{-\frac{1}{n}} \phi^{\prime}(r) t}{x_{0}}\right)^{-\frac{n}{n+1}}\right)\right) t}{\phi\left(r_{-}\right)+n r_{-} \phi^{\prime}\left(r_{-}\right)} .
\end{array}\right.
$$

The propagation speeds of $J_{6}$ and $J_{1}$ are $\tau_{6}=\tau_{1}=\phi\left(r_{-}\right)$. In other words, the two contact discontinuities $J_{1}$ and $J_{6}$ are parallel to each other. In the same way, we can also see that $\xi_{7}\left(u_{2}, v_{2}\right)=\xi_{4}\left(u_{2}, v_{2}\right)=\phi\left(r_{2}\right)+n r_{2} \phi^{\prime}\left(r_{2}\right)=\phi\left(r_{m}\right)+n r_{m} \phi^{\prime}\left(r_{m}\right)$ due to the fact $r_{2}=r_{m}$, which means that the wave front of the rarefaction wave $R_{7}$ and the wave back of the rarefaction wave $R_{4}$ share the same propagation speed.

Case $3 J+S$ and $J+R$.

Let us turn to consider the situation that there are a contact discontinuity followed by a shock wave emitting from the initial point $(0,0)$ and a contact discontinuity followed by a rarefaction wave emitting from the initial point $\left(x_{0}, 0\right)$. The occurrence of this case depends on the conditions $0<r_{m}<r_{-}$and $0<r_{m}<r_{+}$. We use $J_{1}, S_{2}$ and $J_{3}, R_{4}$ to denote them respectively. In this case, when the time $t$ is small enough, the solution to the double Riemann problem (1.1) and (1.6) can be expressed clearly as follows (see Figs. 3 and 4):

$$
\left(u_{-}, v_{-}\right)+J_{1}+\left(u_{1}, v_{1}\right)+S_{2}+\left(u_{m}, v_{m}\right)+J_{3}+\left(u_{2}, v_{2}\right)+R_{4}+\left(u_{+}, v_{+}\right)
$$

The intermediate states $\left(u_{1}, v_{1}\right),\left(u_{2}, v_{2}\right)$, and $\left(u_{3}, v_{3}\right)$ are also the same as those in Case 1. Similarly, the intersection point $\left(x_{1}, t_{1}\right)$ is also the same as that in Case 1. As before, we
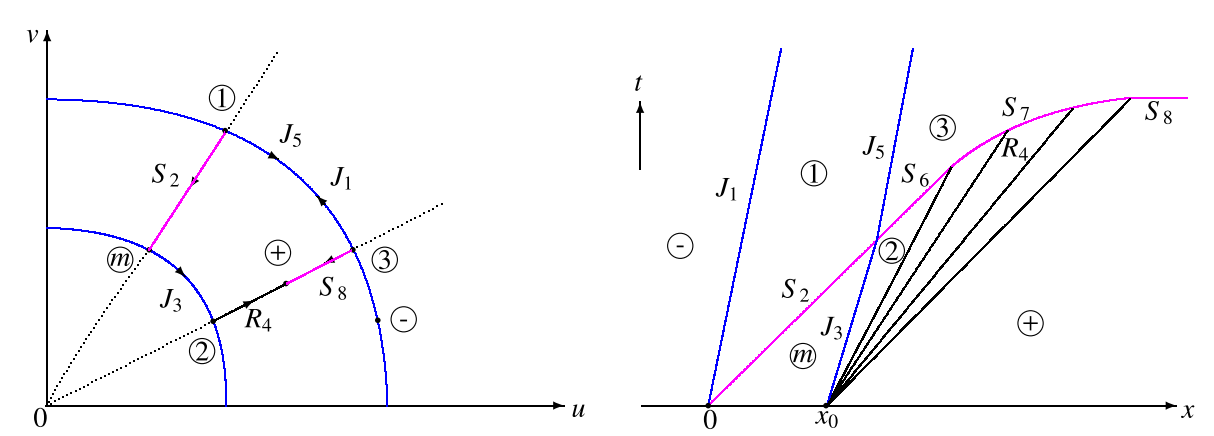

Figure 3 The interaction between $J+S$ and $J+R$ is displayed when $0<r_{m}<r_{+}<r_{-}$, in which the shock wave $S_{6}$ is able to penetrate the whole rarefaction wave $R_{4}$ completely 

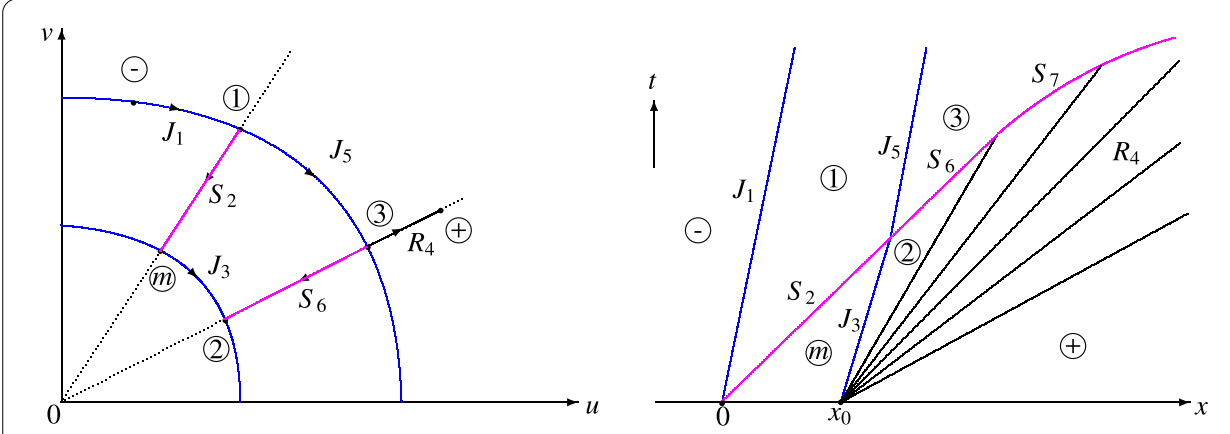

Figure 4 The interaction between $J+S$ and $J+R$ is displayed when $0<r_{m}<r_{-}<r_{+}$, in which the shock wave $S_{6}$ cannot penetrate $R_{4}$ completely and ultimately has the line $x=\left(\phi\left(r_{-}\right)+n r_{-} \phi^{\prime}\left(r_{-}\right)\right) t+x_{0}$ as its asymptote

again have a local Riemann problem at the point $\left(x_{1}, t_{1}\right)$ for system (1.1) subject to the left state $\left(u_{1}, v_{1}\right)$ and the right state $\left(u_{2}, v_{2}\right)$. Due to the relations $r_{2}=r_{m}<r_{1}=r_{-}$, the solution to the local Riemann problem at the point $\left(x_{1}, t_{1}\right)$ is still a contact discontinuity followed by a shock wave, which are denoted by $J_{5}$ and $S_{6}$, respectively. As in those in Case 1, it can be obtained that $\tau_{1}=\tau_{5}$ and $\sigma_{2}=\sigma_{6}$. That is to say, $J_{1}$ is parallel to $J_{5}$ as well as the propagation speed of shock wave is invariant when across the contact discontinuity. In the following, we shall draw our attention to how the shock wave $S_{6}$ penetrates the rarefaction wave $R_{4}$.

Lemma 3.4 The shock wave $S_{6}$ keeps up with the wave back of the rarefaction wave $R_{4}$ in finite time. More specifically, if $r_{+}<r_{-}$, then $S_{6}$ is able to penetrate $R_{4}$ completely. Otherwise, if $r_{+}>r_{-}$, then $S_{6}$ cannot penetrate $R_{4}$ completely and finally takes the line $x=\left(\phi\left(r_{-}\right)+\right.$ $\left.n r_{-} \phi^{\prime}\left(r_{-}\right)\right) t+x_{0}$ as its asymptote.

Proof It suffices to get the propagation speeds of the wave back of the rarefaction wave $R_{4}$ and the shock wave $S_{6}$, which are given respectively by

$$
\xi_{4}\left(u_{2}, v_{2}\right)=\xi_{4}\left(u_{m}, v_{m}\right)=\phi\left(r_{m}\right)+n r_{m} \phi^{\prime}\left(r_{m}\right), \quad \sigma_{6}=\frac{\sqrt[n]{r_{m}} \phi\left(r_{m}\right)-\sqrt[n]{r_{-}} \phi\left(r_{-}\right)}{\sqrt[n]{r_{m}}-\sqrt[n]{r_{-}}}
$$

Hence, we get

$$
\begin{aligned}
\sigma_{6}-\xi_{4}\left(u_{m}, v_{m}\right) & =\frac{\sqrt[n]{r_{m}} \phi\left(r_{m}\right)-\sqrt[n]{r_{-}} \phi\left(r_{-}\right)}{\sqrt[n]{r_{m}}-\sqrt[n]{r_{-}}}-\left(\phi\left(r_{m}\right)+n r_{m} \phi^{\prime}\left(r_{m}\right)\right) \\
& =\sqrt[n]{r_{-}} \frac{\phi\left(r_{-}\right)-\phi\left(r_{m}\right)}{\sqrt[n]{r_{-}}-\sqrt[n]{r_{m}}}-n r_{m} \phi^{\prime}\left(r_{m}\right)
\end{aligned}
$$

By using the mean value theorem again, there exists $\bar{r}_{4} \in\left(r_{m}, r_{-}\right)$such that we have

$$
\sigma_{6}-\xi_{4}\left(u_{m}, v_{m}\right)=n r_{\underline{n}}^{\frac{1}{n}} \overline{r_{4}} \frac{n-1}{n} \phi^{\prime}\left(\bar{r}_{4}\right)-n r_{m} \phi^{\prime}\left(r_{m}\right)>n \overline{r_{4}} \phi^{\prime}\left(\overline{r_{4}}\right)-n r_{m} \phi^{\prime}\left(r_{m}\right) .
$$

It is worthwhile to notice that $r_{4}>r_{m}>0$, which implies that $\phi^{\prime}\left(\overline{r_{4}}\right)>\phi^{\prime}\left(r_{m}\right)>0$ under the assumptions $\phi^{\prime \prime}>0$ and $\phi^{\prime}>0$. Thus, we have $\sigma_{6}>\xi_{4}\left(u_{m}, v_{m}\right)$. In other words, the shock wave $S_{6}$ catches up with the wave back of the rarefaction wave $R_{4}$ in finite time. 
The intersection point $\left(x_{2}, t_{2}\right)$ is determined by

$$
\left\{\begin{array}{l}
x_{2}=\sigma_{6} t_{2}=\frac{\sqrt[n]{r_{m}} \phi\left(r_{m}\right)-\sqrt[n]{r_{-}} \phi\left(r_{-}\right)}{\sqrt[n]{r_{m}}-\sqrt[n]{r_{-}}} t_{2} \\
x_{2}-x_{0}=\xi_{4}\left(u_{m}, v_{m}\right) t_{2}=\left(\phi\left(r_{m}\right)+n r_{m} \phi^{\prime}\left(r_{m}\right)\right) t_{2}
\end{array}\right.
$$

which yields

$$
\begin{aligned}
\left(x_{2}, t_{2}\right)= & \left(\frac{x_{0}\left(\sqrt[n]{r_{m}} \phi\left(r_{m}\right)-\sqrt[n]{r_{-}} \phi\left(r_{-}\right)\right)}{\sqrt[n]{r_{-}}\left(\phi\left(r_{m}\right)-\phi\left(r_{-}\right)\right)-n r_{m} \phi^{\prime}\left(r_{m}\right)\left(\sqrt[n]{r_{m}}-\sqrt[n]{r_{-}}\right)},\right. \\
& \left.\frac{x_{0}\left(\sqrt[n]{r_{m}}-\sqrt[n]{r_{-}}\right)}{\sqrt[n]{r_{-}}\left(\phi\left(r_{m}\right)-\phi\left(r_{-}\right)\right)-n r_{m} \phi^{\prime}\left(r_{m}\right)\left(\sqrt[n]{r_{m}}-\sqrt[n]{r_{-}}\right)}\right) .
\end{aligned}
$$

The interaction between the wave back of the rarefaction wave $R_{4}$ and the shock wave $S_{6}$ occurs at the point $\left(x_{2}, t_{2}\right)$, where we again have a local Riemann problem for system (1.1). The shock wave $S_{6}$ begins to penetrate the rarefaction wave $R_{4}$ after $t_{2}$, which is recorded as $S_{7}$ during the process of penetration.

The expression for $S_{7}$ during the process of penetration is calculated by

$$
\left\{\begin{array}{l}
\frac{d x}{d t}=\frac{\sqrt[n]{r} \phi(r)-\sqrt[n]{r_{-}} \phi\left(r_{-}\right)}{\sqrt[n]{r}-\sqrt[n]{r_{-}}}, \\
\frac{x-x_{0}}{t}=\phi(r)+n r \phi^{\prime}(r), \\
x\left(t_{2}\right)=x_{2}
\end{array}\right.
$$

in which $r$ varies from $r_{2}\left(=r_{m}\right)$ to $r_{+}$. Depending on the ordering relation between $r_{+}$and $r_{-}$, there are two possible situations to occur during the process of penetration as follows:

(1) If $r_{+}<r_{-}$, then the shock wave $S_{6}$ is able to penetrate the whole rarefaction wave $R_{4}$ completely in finite time and subsequently forms a new shock wave $S_{8}$ (see Fig. 3), whose propagation speed is given by $\sigma_{8}=\frac{\sqrt[n]{r_{+}} \phi\left(r_{+}\right)-\sqrt[n]{r_{-}} \phi\left(r_{-}\right)}{\sqrt[n]{r_{+}}-\sqrt[n]{r_{-}}}$.

(2) If $r_{+}>r_{-}$, then the shock wave $S_{6}$ cannot penetrate $R_{4}$ completely in finite time and ultimately has the line $x=\left(\phi\left(r_{-}\right)+n r_{-} \phi^{\prime}\left(r_{-}\right)\right) t+x_{0}$ as its asymptote (see Fig. 4).

Case $4 J+R$ and $J+S$.

In the end, we mainly consider the situation that there are a contact discontinuity followed by a rarefaction wave emitting from the initial point $(0,0)$ and a contact discontinuity followed by a shock wave emitting from the initial point $\left(x_{0}, 0\right)$. The occurrence of this case depends on the conditions $0<r_{-}<r_{m}$ and $0<r_{+}<r_{m}$. Moreover, we use $J_{1}, R_{2}$ and $J_{3}$, $S_{4}$ to denote them respectively (see Fig. 5). In this case, when the time $t$ is small enough, the solution to the double Riemann problem (1.1) and (1.6) may be expressed simply as follows (see Figs. 5 and 6):

$$
\left(u_{-}, v_{-}\right)+J_{1}+\left(u_{1}, v_{1}\right)+R_{2}+\left(u_{m}, v_{m}\right)+J_{3}+\left(u_{2}, v_{2}\right)+S_{4}+\left(u_{+}, v_{+}\right)
$$

Here and below the intermediate states $\left(u_{1}, v_{1}\right),\left(u_{2}, v_{2}\right)$, and $\left(u_{3}, v_{3}\right)$ and all the situations before the time $t_{1}$ are the same as those in Case 2 . Thus, we shall draw our attention to the process of the shock wave $S_{4}$ penetrating the rarefaction wave $R_{7}$. 

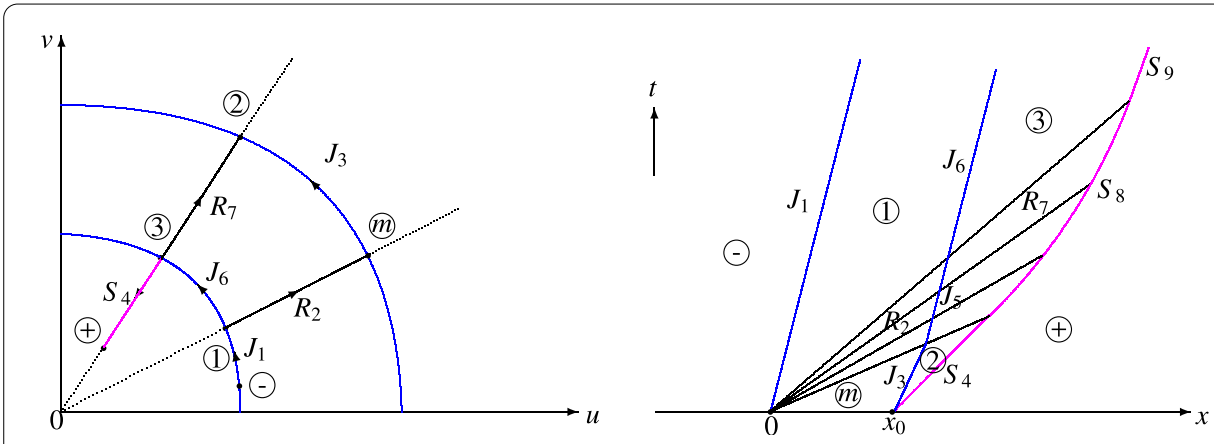

Figure 5 The interaction between $J+R$ and $J+S$ is shown when $0<r_{+}<r_{-}<r_{m}$, in which the shock wave $S_{4}$ is able to penetrate the rarefaction wave $R_{7}$ completely in finite time
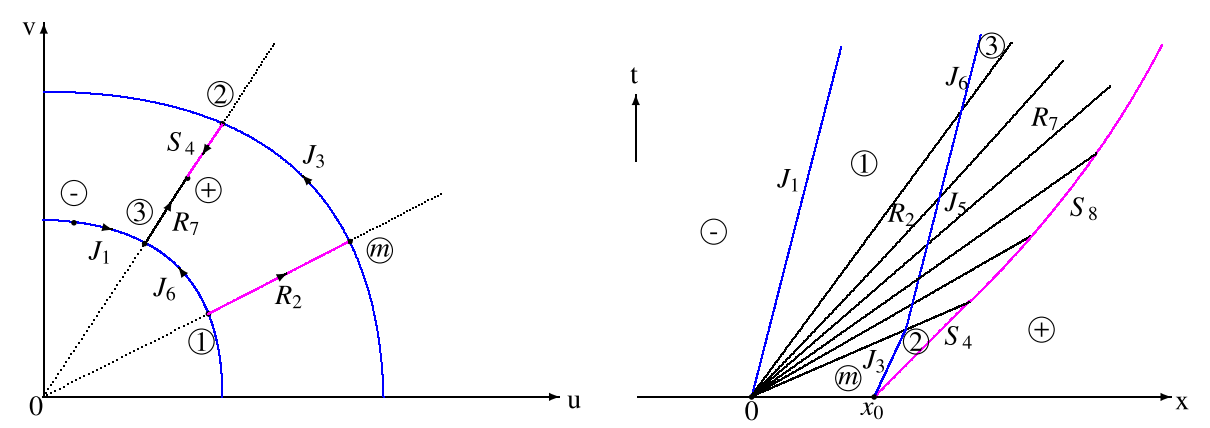

Figure 6 The interaction between $J+R$ and $J+S$ is shown when $0<r_{-}<r_{+}<r_{m}$, in which the shock wave $S_{4}$ cannot penetrate the rarefaction wave $R_{7}$ completely and ultimately has the line $x=\left(\phi\left(r_{+}\right)+n r_{+} \phi^{\prime}\left(r_{+}\right)\right) t$ as its asymptote

Lemma 3.5 The wave front of the rarefaction wave $R_{7}$ catches up with the shock wave $S_{4}$ in finite time. More specifically, if $r_{+}<r_{-}$, then $S_{4}$ is able to penetrate $R_{7}$ completely. Otherwise, if $r_{+}>r_{-}$, then $S_{4}$ cannot penetrate $R_{7}$ completely and ultimately has the line $x=\left(\phi\left(r_{+}\right)+n r_{+} \phi^{\prime}\left(r_{+}\right)\right)$t as its asymptote.

Proof It is easily shown that the propagation speeds of the wave fronts of $R_{7}$ and $S_{4}$ are given respectively by

$$
\xi_{7}\left(u_{2}, v_{2}\right)=\xi_{7}\left(u_{m}, v_{m}\right)=\phi\left(r_{m}\right)+n r_{m} \phi^{\prime}\left(r_{m}\right), \quad \sigma_{4}=\frac{\sqrt[n]{r_{+}} \phi\left(r_{+}\right)-\sqrt[n]{r_{m}} \phi\left(r_{m}\right)}{\sqrt[n]{r_{+}}-\sqrt[n]{r_{m}}}
$$

Thus, we get

$$
\begin{aligned}
\sigma_{4}-\xi_{7}\left(u_{m}, v_{m}\right) & =\frac{\sqrt[n]{r_{+}} \phi\left(r_{+}\right)-\sqrt[n]{r_{m}} \phi\left(r_{m}\right)}{\sqrt[n]{r_{+}}-\sqrt[n]{r_{m}}}-\left(\phi\left(r_{m}\right)+n r_{m} \phi^{\prime}\left(r_{m}\right)\right) \\
& =\sqrt[n]{r_{+}} \frac{\phi\left(r_{+}\right)-\phi\left(r_{m}\right)}{\sqrt[n]{r_{+}}-\sqrt[n]{r_{m}}}-n r_{m} \phi^{\prime}\left(r_{m}\right)
\end{aligned}
$$

With the similar method as before, there exists $\overline{r_{5}} \in\left(r_{+}, r_{m}\right)$ such that one also has

$$
\sigma_{4}-\xi_{7}\left(u_{m}, v_{m}\right)=n r_{+}^{\frac{1}{n}} \bar{r}_{5}^{\frac{n-1}{n}} \phi^{\prime}\left(\bar{r}_{5}\right)-n r_{m} \phi^{\prime}\left(r_{m}\right)<n \overline{r_{5}} \phi^{\prime}\left(\bar{r}_{5}\right)-n r_{m} \phi^{\prime}\left(r_{m}\right) .
$$


It is obvious to see that $0<\overline{r_{5}}<r_{m}$, which implies that $0<\phi^{\prime}\left(\overline{r_{5}}\right)<\phi^{\prime}\left(r_{m}\right)$ under the assumptions $\phi^{\prime \prime}>0$ and $\phi^{\prime}>0$. Thus, it can be concluded that $\sigma_{4}<\xi_{7}\left(u_{m}, v_{m}\right)$. In other words, the wave front of the rarefaction wave $R_{7}$ catches up with the shock wave $S_{4}$ in finite time. The intersection point $\left(x_{3}, t_{3}\right)$ can be calculated by

$$
\left\{\begin{array}{l}
x_{3}=\xi_{7}\left(u_{m}, v_{m}\right) t_{3}=\phi\left(r_{m}\right)+n r_{m} \phi^{\prime}\left(r_{m}\right) t_{3} \\
x_{3}-x_{0}=\sigma_{4} t_{3}=\frac{\sqrt[n]{r_{+}} \phi\left(r_{+}\right)-\sqrt[n]{r_{m}} \phi\left(r_{m}\right)}{\sqrt[n]{r_{+}}-\sqrt[n]{r_{m}}} t_{3}
\end{array}\right.
$$

Thus, we have

$$
\begin{aligned}
\left(x_{3}, t_{3}\right)= & \left(\frac{x_{0}\left(\sqrt[n]{r_{+}}-\sqrt[n]{r_{m}}\right)\left(\phi\left(r_{m}\right)+n r_{m} \phi^{\prime}\left(r_{m}\right)\right)}{n r_{m} \phi^{\prime}\left(r_{m}\right)\left(\sqrt[n]{r_{+}}-\sqrt[n]{r_{m}}\right)-\sqrt[n]{r_{+}}\left(\phi\left(r_{+}\right)-\phi\left(r_{m}\right)\right)},\right. \\
& \left.\frac{x_{0}\left(\sqrt[n]{r_{+}}-\sqrt[n]{r_{m}}\right)}{n r_{m} \phi^{\prime}\left(r_{m}\right)\left(\sqrt[n]{r_{+}}-\sqrt[n]{r_{m}}\right)-\sqrt[n]{r_{+}}\left(\phi\left(r_{+}\right)-\phi\left(r_{m}\right)\right)}\right) .
\end{aligned}
$$

It is clear to see that the shock wave $S_{4}$ penetrates the rarefaction wave $R_{7}$ with the varying propagation speed after the time $t_{3}$. During the process of penetration, the curve of $S_{8}$ is determined by

$$
\left\{\begin{array}{l}
\frac{d x}{d t}=\frac{\sqrt[n]{r_{+}} \phi\left(r_{+}\right)-\sqrt[n]{r} \phi(r)}{\sqrt[n]{r_{+}}-\sqrt[n]{r}}, \\
\frac{x}{t}=\phi(r)+n r \phi^{\prime}(r), \\
x\left(t_{3}\right)=x_{3}
\end{array}\right.
$$

in which $r$ varies from $r_{2}\left(=r_{m}\right)$ to $r_{3}\left(=r_{1}=r_{-}\right)$. Similar to those in Case 3 , there are also two possible situations in the process of penetration as follows:

(1) If $r_{+}<r_{-}$, then the shock wave $S_{4}$ is able to penetrate the rarefaction wave $R_{7}$ completely in finite time and subsequently forms a new shock wave $S_{9}$ (see Fig. 5), whose propagation speed is given by $\sigma_{9}=\frac{\sqrt[n]{r_{+}} \phi\left(r_{+}\right)-\sqrt[n]{r_{-}} \phi\left(r_{-}\right)}{\sqrt[n]{r_{+}}-\sqrt[n]{r_{-}}}$.

(2) If $r_{+}>r_{-}$, then the shock wave $S_{4}$ cannot penetrate the rarefaction wave $R_{7}$ completely in finite time and ultimately has the line $x=\left(\phi\left(r_{+}\right)+n r_{+} \phi^{\prime}\left(r_{+}\right)\right) t$ as its asymptote (see Fig. 6).

Up to now, we have finished the discussions on all kinds of wave interactions for system (1.1) under our assumptions $\phi^{\prime \prime}(r)>0$ and $\phi^{\prime}(r)>0$. The global solutions to the double Riemann problem (1.1) and (1.6) are constructed completely in explicit forms for all kinds of situations. It can be seen clearly from the above cases that the main conclusion of this paper can be drawn as follows:

Theorem 3.6 If the perturbed parameter $x_{0}$ tends to zero, then the global solutions to the double Riemann problem (1.1) and (1.6) are exactly identical with those to the Riemann problem (1.1) and (1.7) for the same Riemann initial data. Thus, it turns out that the solutions to the Riemann problem (1.1) and (1.7) are stable with respect to the specific small perturbation in the form (1.6) of the Riemann initial data (1.7). 


\section{Competing interests}

The authors declare that they have no competing interests.

\section{Authors' contributions}

The authors contributed equally and significantly in writing this article. All authors read and approved the final manuscript.

\section{Author details}

'School of Mathematics and Statistics Science, Ludong University, Yantai, P.R. China. ${ }^{2}$ School of Mathematical Sciences, Qufu Normal University, Qufu, P.R. China.

\section{Publisher's Note}

Springer Nature remains neutral with regard to jurisdictional claims in published maps and institutional affiliations.

Received: 1 November 2018 Accepted: 13 February 2019 Published online: 21 February 2019

\section{References}

1. Chen, G.Q.: Hyperbolic systems of conservation laws with a symmetry. Commun. Partial Differ. Equ. 16, 1461-1487 (1991)

2. Freistuhler, H.: Rotational degeneracy of hyperbolic systems of conservation laws. Arch. Ration. Mech. Anal. 113, 39-64 (1990)

3. Lu, Y.G.: Existence of global entropy solutions to general system of Keyfitz-Kranzer type. J. Funct. Anal. 264, 2457-2468 (2013)

4. Shen, C.: Delta shock wave solution for a symmetric Keyfitz-Kranzer system. Appl. Math. Lett. 77, 35-43 (2018)

5. Hernandez, J.C.: Existence of weak entropy solution for a symmetric system of Keyfitz-Kranzer type. Rev. Colomb. Mat. 47, 13-28 (2013)

6. Temple, B.: Systems of conservation laws with coinciding shock and rarefaction cures. Contemp. Math. 17, 143-151 (1983)

7. Temple, B.: Systems of conservation laws with invariant submanifolds. Trans. Am. Math. Soc. 280, 781-795 (1983)

8. Keyfitz, B.L., Kranzer, H.C.: A system of nonstrictly hyperbolic conservation laws arising in elasticity. Arch. Ration. Mech. Anal. 72, 219-241 (1980)

9. Kearsley, A., Reiff, A.: Existence of weak solutions to a class of nonstrictly hyperbolic conservation laws with non-interacting waves. Pac. J. Math. 205, 153-170 (2002)

10. Shen, C.: Wave interactions and stability of the Riemann solutions for the chromatography equations. J. Math. Anal. Appl. 365, 609-618 (2010)

11. Guo, L., Pan, L., Yin, G.: The perturbed Riemann problem and delta contact discontinuity in chromatography equations. Nonlinear Anal. TMA 106, 110-123 (2014)

12. Sun, M.: Interactions of delta shock waves for the chromatography equations. Appl. Math. Lett. 26, 631-637 (2013)

13. Shen, $\mathrm{C}$ : The asymptotic behaviors of solutions to the perturbed Riemann problem near the singular curve for the chromatography system. J. Nonlinear Math. Phys. 22, 76-101 (2015)

14. Wang, G.: One-dimensional non-linear chromatography system and $\delta$-shock waves. Z. Angew. Math. Phys. 64 , $1451-1469(2013)$

15. Yang, H., Zhang, Y.: New developments of delta shock waves and its applications in systems of conservation laws. J. Differ. Equ. 252, 5951-5993 (2012)

16. Lu, Y.G.: Existence of global bounded weak solutions to a non-symmetric system of Keyfitz-Kranzer type. J. Funct. Anal. 261, 2797-2815 (2011)

17. Lu, Y.G.: Existence of global bounded weak solutions to a symmetric system of Keyfitz-Kranzer type. Nonlinear Anal., Real World Appl. 13, 235-240 (2012)

18. Sheng, W., Zhang, T.: The Riemann problem for the transportation equations in gas dynamics. Mem. Am. Math. Soc. 137, N654 (1999)

19. Shen, C., Sun, M.: A distributional product approach to the delta shock wave solution for the one-dimensional zero-pressure gas dynamics system. Int. J. Non-Linear Mech. 105, 105-122 (2018)

20. Lai, G., Sheng, W.: Elementary wave interactions to the compressible Euler equations for Chaplygin gas in two dimensions. SIAM J. Appl. Math. 76, 2218-2242 (2016)

21. Lai, G., Sheng, W., Zheng, Y.: Simple waves and pressure delta waves for a Chaplygin gas in multi-dimensions. Discrete Contin. Dyn. Syst. 31, 489-523 (2011)

22. Tong, M., Shen, C., Lin, X.: The asymptotic limits of Riemann solutions for the isentropic extended Chaplygin gas dynamic system with the vanishing pressure. Bound. Value Probl. 2018, Article ID 144 (2018)

23. Guo, L., Zhang, Y., Yin, G.: Interactions of delta shock waves for the Chaplygin gas equations with split delta functions. J. Math. Anal. Appl. 410, 190-201 (2014)

24. Shen, C.: The Riemann problem for the Chaplygin gas equations with a source term. Z. Angew. Math. Mech. 96 , 681-695 (2016)

25. Sun, M.: Singular solutions to the Riemann problem for a macroscopic production model. Z. Angew. Math. Mech. 97, 916-931 (2017)

26. Chang, T., Hsiao, L.: The Riemann Problem and Interaction of Waves in Gas Dynamics. Pitman Monographs and Surveys in Pure and Applied Mathematics, vol. 41. Longman, Harlow (1989)

27. Raja Sekhar, T., Sharma, V.D.: Riemann problem and elementary wave interactions in isentropic magnetogasdynamics. Nonlinear Anal., Real World Appl. 11, 619-636 (2010)

28. Raja Sekhar, T., Sharma, V.D.: Interaction of shallow water waves. Stud. Appl. Math. 121, 1-25 (2008)

29. Sun, M.: Interactions of elementary waves for Aw-Rascle model. SIAM J. Appl. Math. 69, 1542-1558 (2009) 
30. Wei, X., Shen, C.: Structural stability of Riemann solutions for strictly hyperbolic systems with three piecewise constant states. Electron. J. Differ. Equ. 2016, Article ID 320 (2016)

31. Hu, Y., Wang, G.: The interaction of rarefaction waves of a two-dimensional nonlinear wave system. Nonlinear Anal., Real World Appl. 22, 1-15 (2015)

32. Abdelrahman, M.A.E., Kunik, M.: The interaction of waves for the ultra-relativistic Euler equations. J. Math. Anal. Appl. 409, 1140-1158 (2014)

33. Xu, Y., Wang, L.: Breakdown of classical solutions to Cauchy problem for inhomogeneous quasilinear hyperbolic systems. Indian J. Pure Appl. Math. 46, 827-851 (2015)

34. Lin, X., Zhao, Z.: Iterative technique for a third-order differential equation with three-point nonlinear boundary value conditions. Electron. J. Qual. Theory Differ. Equ. 2016, Article ID 12 (2016)

35. Lin, X., Zhao, Z.: Existence and uniqueness of symmetric positive solutions of $2 n$-order nonlinear singular boundary value problems. Appl. Math. Lett. 26, 692-698 (2013)

Submit your manuscript to a SpringerOpen ${ }^{0}$ journal and benefit from:

- Convenient online submission

- Rigorous peer review

Open access: articles freely available online

- High visibility within the field

- Retaining the copyright to your article

Submit your next manuscript at $\boldsymbol{\nabla}$ springeropen.com 